\title{
Combination Product
}

\author{
National Cancer Institute
}

\section{Source}

National Cancer Institute. Combination Product. NCI Thesaurus. Code C54696.

A product comprised of two or more regulated components, i.e., drug and device, biologic and device, that are physically, chemically, or otherwise combined or mixed and produced as a single entity; or two or more separate products packaged together in a single package or as a unit and comprised of drug and device products, device and biological products, or biological and drug products; or a drug, device, or biological product packaged separately that according to its investigational plan or proposed labeling is intended for use only with an approved individually specified drug, device, or biological product where both are required to achieve the intended use, indication, or effect and where upon approval of the proposed product the labeling of the approved product would need to be changed, e.g., to reflect a change in intended use, dosage form, strength, route of administration, or significant change in dose; or any investigational drug, device, or biological product packaged separately that according to its proposed labeling is for use only with another individually specified investigational drug, device, or biological product where both are required to achieve the intended use, indication, or effect. 